Divers@ Revista Eletrônica Interdisciplinar,

Matinhos, v. 13, n. 1, p. 60-67, jan./jun. 2020

ISSN 1983-8921

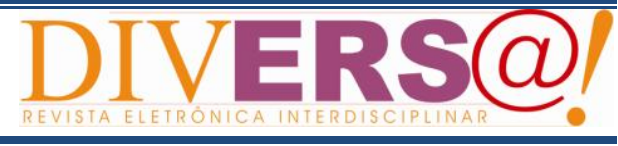

\title{
ANÁLISE DO PERFIL DE DOCENTES USANDO DADOS COLETADOS DA PLATAFORMA LATTES
}

\section{PROFILE ANALYSIS OF PROFESSORS USING DATA COLLECTED FROM THE LATTES PLATFORM}

Ernani Viriato de Melo ${ }^{1}$

Rafhael Menezes Braga ${ }^{2}$

\begin{abstract}
Resumo
A pesquisa acadêmica é extremamente importante para a discussão e difusão de conhecimento e é amplamente incentivada dentro dos Institutos e Universidades Federais. Para o sucesso da pesquisa, o discente muitas vezes precisa de uma contribuição de pesquisadores, da própria instituição, que sejam especialistas em determinados assuntos, entretanto encontrá-los pode ser uma tarefa árdua. Para mitigar essa limitação, este trabalho propõe uma plataforma onde o aluno poderá encontrar informações sobre o perfil dos docentes do Instituto Federal do Triângulo Mineiro (IFTM). Para mapear o perfil docente, foram coletados os currículos Lattes de 544 docentes do IFTM no mês de dezembro de 2018. Ao analisar a formação acadêmica destes docentes, percebe-se que as formações com maior número de docentes são: 66 em Letras, 54 em Agronomia, 48 em Engenharia Elétrica, 46 em Ciência da Computação, 43 em Administração, 39 em Matemática, 37 em Química e 35 em Ciências Biológicas. Analisando a produção científica de 2014 a 2018, percebe-se que as palavras-chave mais usadas no IFTM são: avaliação; ensino e aprendizagem; soja; cerrado; produtividade; educação; irrigação; plantas; ciclagem de nutrientes; resíduos; cultivo; milho; alface; solo; ensino médio; qualidade; professores; leite; química; consumo; água; cultura; dentre outras.
\end{abstract}

Palavras-chave: Formação docente; Pesquisa acadêmica; IFTM.

\begin{abstract}
Academic research is extremely important for knowledge discussion and dissemination and is widely encouraged within Federal Institutes and Universities. For the success of the research, the student often needs a contribution from researchers from the institution, who are experts in certain subjects, but finding them can be an arduous task. To mitigate this limitation, this work proposes a platform where the student can find information about the profile of the professors of the Instituto Federal do Triangulo Mineiro (IFTM). In order to map the teaching profile, the curriculum of 544 professors of IFTM were collected in December 2018. When analyzing the academic training of these professors, it can be seen that the formations with the greatest number of professors are: 66 in Letters, 54 in Agronomy, 48 in Electrical Engineering, 46 in Computer Science, 43 in Administration, 39 in Mathematics, 37 in Chemistry and 35 in Biological Sciences. Analyzing scientific production from 2014 to 2018, it can be seen that the keywords most frequently used in IFTM are: evaluation; teaching and learning; Soy; thick; productivity; education; irrigation; plants; nutrient cycling; waste; cultivation; corn; lettuce; ground; high school; quality; professors; milk; chemistry; consumption; Water; culture; among others.
\end{abstract}

Keywords: Teacher Training; Academic Research; IFTM.

\footnotetext{
Artigo Original: Recebido em 10/09/2019 - Aprovado em 10/06/2020

${ }^{1}$ Doutor em Ciência da Computação, Docente do Instituto Federal do Triângulo Mineiro. e-mail: ernanimelo@iftm.edu.br (autor correspondente)

2 Discente do curso de Engenharia da Computação, Instituto Federal do Triângulo Mineiro. e-mail: rafhaelmbraga@gmail.com
} 


\section{Introdução}

O Instituto Federal de Educação, Ciência e Tecnologia do Triângulo Mineiro (IFTM), em consonância com a Lei 11.892 , de 29 de dezembro de 2008, foi criado mediante integração dos antigos Centros Federais de Educação Tecnológica, Escolas Técnicas e Agrotécnicas e define-se como uma "instituição de educação superior, básica e profissional, pluricurricular e multicampi" (IFTM, 2019b). O IFTM é composto pelos campi Campina Verde, Ituiutaba, Paracatu, Patos de Minas, Patrocínio, Uberaba, Uberaba Parque Tecnológico, Uberlândia e Uberlândia Centro e pela Reitoria.

O tripé formado pelo ensino, pesquisa e extensão constitui o eixo fundamental do IFTM. O ensino, a pesquisa e a extensão, enquanto atividades complementares e interdependentes, precisam ter valorações equivalentes na Rede Federal de Educação Profissional, Científica e Tecnológica (RFEPCT), sob o risco de desenvolver conhecimento mutilante e reducionista (FORPROEX, 2006).

As atividades de pesquisa do IFTM são gerenciadas pela Pró-Reitoria de Pesquisa, PósGraduação e Inovação (PROPI). O IFTM possui diversos programas de bolsas de iniciação científica e inovação tecnológica e um programa de iniciação científica voluntária para estudantes. As bolsas são financiadas pelo Conselho Nacional de Desenvolvimento Científico e Tecnológico (CNPq), pela Fapemig e pelo próprio IFTM (IFTM, 2019a). Os programas são: PIBIC, PIBITI, PIBIC-EM E BICJúnior e PIVIC.

O Programa Institucional de Bolsas de Iniciação Científica (PIBIC) visa estimular o pensamento científico e iniciação à pesquisa de estudantes de graduação. O Programa Institucional de Bolsas de Iniciação em Desenvolvimento Tecnológico e Inovação (PIBITI) tem por objetivo estimular os jovens do ensino superior nas atividades, metodologias, conhecimentos e práticas próprias ao desenvolvimento tecnológico e processos de inovação (CNPq, 2019).

O Programa Institucional de Bolsas de Iniciação Científica para o Ensino Médio (PIBIC-EM) e o BICJúnior têm por finalidade incentivar os alunos do
Ensino Médio Integrado ao Técnico (EMI) a realizarem atividades de iniciação científica, desenvolvendo atitudes, habilidades e valores necessários à educação científica (CNPq, 2019).

O Programa Institucional Voluntário de Iniciação Científica (PIVIC) é fundamental para o desenvolvimento científico da RFEPCT, devido à escassez do número de bolsas. Este programa tem por objetivo estimular os pesquisadores produtivos a engajarem os estudantes de graduação e EMI em projetos de pesquisa, sem a concessão de bolsas.

É extremamente importante para o desenvolvimento econômico e social de um país o desenvolvimento de pesquisas básicas e aplicadas:
[...] para desenvolver um país é necessário desenvolver pessoas: elevar o patamar de informação disponível e prover a população de conhecimentos básicos de ciência e tecnologia [...]. Além disso, é necessário estimular os jovens a se tornarem profissionais da ciência e da tecnologia, para avançarmos no conhecimento existente. Assim, é preciso que desde os primeiros anos da educação formal os (as) estudantes sejam postos em contato com a cultura científica [...]. (CNPQ, 2019).

Vale ressaltar que a qualidade no processo de ensino e aprendizagem e a aproximação entre as instituições de ensino e a sociedade está relacionada com o princípio da indissociabilidade entre ensino, pesquisa e extensão:

Pesquisa como princípio científico e
educativo faz parte de todo processo
emancipatório, no qual se constrói o sujeito
histórico autossuficiente, crítico e
autocrítico, participante e capaz de reagir
contra a situação de objeto e de não
cultivar o outro como objeto [...] Pesquisa
como diálogo é processo cotidiano
integrante do ritmo de vida, produto e motivo
de interesses sociais em confronto, base da
aprendizagem que não se restrinja a mera
reprodução; na acepção mais simples, pode
significar conhecer, saber, informar-se para
sobreviver, para enfrentar a vida de modo
consciente. (DEMO, 2011. p.43).

Segundo Pedro Demo (2011), é importante compreender que sem pesquisa não há ensino e que a pesquisa é um satisfatório instrumento para a apropriação, construção e renovação do conhecimento. 
Destarte, alguns trabalhos descritos na literatura utilizaram a Plataforma Lattes ${ }^{\mathrm{i}}$ para gerar informações preciosas e auxiliar no diagnóstico da pesquisa acadêmica em determinadas instituições. Oliveira, Bermejo e Kern (2014) descrevem uma ferramenta desktop, denominada GeraLattes, que constrói informações gerenciais e foi utilizada pela UNIVALI. Digiampietri et al. (2015), descreve um banco de dados formado por mais de um milhão de currículos minerados da Plataforma Lattes que foram processados, organizados e analisados para servirem de base para a produção e análise de redes sociais de pesquisa.

A Coordenadoria de Transferência e Inovação Tecnológica (CTIT) da UFMG disponibiliza uma plataforma, denominada "Somos", para facilitar o mapeamento das competências dos pesquisadores, suas especialidades e produção científica, além de informações sobre departamentos e ativos de propriedade intelectual (SOMOS-UFMG, 2020). Todas as informações apresentadas na plataforma, com exceção dos dados sobre laboratórios e propriedade intelectual, são retiradas da Plataforma Lattes. Além da UFMG (somos.ufmg.br), outras instituições também utilizam esta plataforma, tais como: UFU (somos.ufu.br) e UNICAMP (somos.unicamp.br).

Alguns trabalhos voltados para a pós-graduação stricto sensu utilizam a ferramenta scriptLattes ${ }^{\text {ii }}$ para análise de produção bibliográfica, auxílio na prestação de contas anual e quadrienal e planejamento de pesquisas (MACCARI; KNIESS; FERRAZ, 2019; NIGRO et al., 2016). Segundo Corrêa et al. (2017) a ferramenta scriptLattes facilita e diminui o tempo para a análise de uma grande quantidade de currículos, geração de indicadores bibliométricos e verificação das redes de interação entre autores e coautores.

Até o presente trabalho, os docentes do IFTM utilizam a Plataforma Lattes para atualizarem suas formações, produções de artigos em eventos e periódicos, produções de livros e capítulos de livros, dentre outras informações. Entretanto, o acesso às informações se dá individualmente, dificultando o trabalho da PROPI na identificação de perfis de pesquisa dos docentes do IFTM e o mapeamento de suas linhas de pesquisa.
Este trabalho tem o objetivo de mapear o perfil de professores e de áreas de pesquisa utilizando a coleta de dados da Plataforma Lattes. Dessa forma, pretende-se estimular o trabalho científico da comunidade acadêmica através da facilidade de contato de pessoas com áreas de interesse semelhante.

\section{Material e métodos}

Para a realização deste trabalho, foi necessário o desenvolvimento de diversas atividades que estão organizadas em 5 etapas, conforme Figura 1.

Figura 1 - Etapas dos Procedimentos Metodológicos

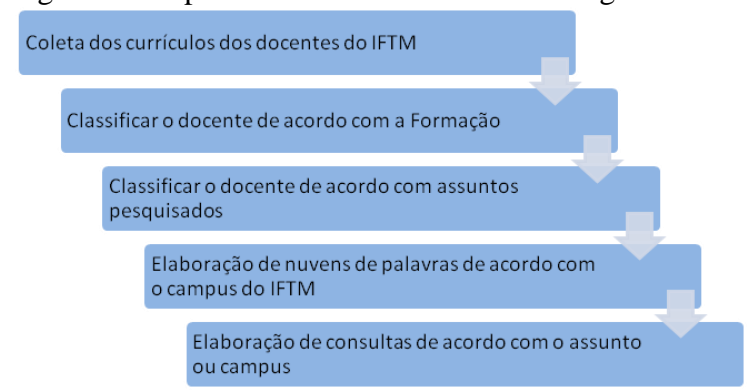

Fonte: Elaborado pelos autores (2020).

\subsection{Coleta dos currículos dos docentes do IFTM}

Nesta etapa do projeto, foi solicitado ao IFTM, usando o Sistema Eletrônico do Serviço de Informação ao Cidadão ${ }^{i i i}$, uma listagem de todos os docentes permanentes do IFTM, separados por campus. Em seguida, foi catalogado o link do currículo Lattes de cada docente. Ao todo, foram catalogados 544 dos 561 docentes permanentes do IFTM.

A Plataforma Lattes apresenta bases de dados de Currículos e Grupos de Pesquisa (CNPQ, 2019). O currículo Lattes contempla várias informações importantes, tais como, formação acadêmica, experiência profissional, projetos de pesquisa, produção técnica e científica, participação em eventos, orientações de iniciação científica e pósgraduação, entre outros, o que torna uma ótima fonte de informações acadêmicas que podem ser coletadas.

O currículo Lattes de cada docente está disponível na página web ou em arquivo XML para 
download. Neste trabalho, realizamos o download dos arquivos XML de todos os docentes no período de dezembro de 2018.

$\mathrm{O}$ arquivo XML do currículo é dividido em tags e de acordo com a tag é identificado a finalidade da informação. $\mathrm{O}$ algoritmo implementado neste trabalho interpreta as tags do arquivo XML e armazena no banco de dados as seguintes informações: Formação; Produção de Artigos em eventos e periódicos, Produção de livros e capítulos de livros e Orientações de iniciação científica e pósgraduação.

\subsection{Classificar o docente de acordo com a Formação}

Após coletada todas as informações referentes a formação acadêmica de todos os docentes, o intuito desta etapa é classificar o professor de acordo com suas graduações de modo a facilitar a pesquisa do perfil docente.

\subsection{Classificar o docente de acordo com os assuntos pesquisados}

Com o propósito de identificar as áreas de pesquisa atuantes no IFTM, foram extraídas as palavras-chave fornecidas pelas publicações de artigos em periódicos e eventos de todos os docentes, criando assim uma base de dados. Em seguida, realizou-se a contagem da frequência dessas palavras-chave para cada docente. Para a realização desta contagem, foi desenvolvido um algoritmo que calcula a ocorrência das palavras nos títulos dos trabalhos publicados de cada docente. Desse modo, é possível identificar quais docentes atuam em determinadas áreas de pesquisa.

\subsection{Elaboração de nuvens de palavras de acordo com o campus do IFTM}

Com a finalidade de identificar as áreas de pesquisa atuantes em cada campus do IFTM, os docentes foram agrupados de acordo com o campus e assim realizou-se a contagem da repetição de palavras-chave de acordo com o campus. Em seguida, foi elaborada uma nuvem de palavras destacando as palavras com maior frequência para cada campus.

\subsection{Elaboração de consultas de acordo com o assunto ou campus}

Para disponibilizar os perfis dos docentes e as áreas de pesquisa atuantes no IFTM, foi desenvolvido uma plataforma web onde o usuário consulta: os assuntos mais pesquisados de acordo com o ano e campus; a formação acadêmica dos docentes de acordo com o campus; e os docentes de acordo com o campus e assunto pesquisado. Para a produção da plataforma web foram utilizados os seguintes frameworks: React ${ }^{\mathrm{iv}}$ para a programação do front-end; Primeface ${ }^{\mathrm{v}}$ e MaterialUI ${ }^{\mathrm{vi}}$ para a programação da interface com o usuário; e Asp Net Core $^{\text {vii }}$ para programação do back-end. A plataforma web está disponível na página do grupo de pesquisa dos autores no link <https://obsiftm.midi.upt.iftm.edu.br> (OBSERVATÓRIO IFTM, 2019).

A partir das etapas concluídas, foi possível gerar informações importantes para diagnosticar os perfis dos docentes e as áreas de pesquisa atuantes no IFTM. Os resultados serão discutidos na próxima seção.

\section{Resultados}

O foco desta seção é discutir os resultados obtidos pelo mapeamento dos perfis dos docentes e áreas de pesquisa do IFTM. Para isso, os resultados são divididos em três subseções: Análise $d a$ frequência de palavras-chave; Análise da formação acadêmica; e Consulta de docentes por assunto.

\subsection{Análise de frequência de palavras-chave}

Nesta primeira etapa, analisou-se no período de 2014 a 2018 a frequência de palavras-chave de acordo com os campi da instituição. A Figura 2 mostra a nuvem de assuntos de todos os docentes do IFTM e a Figura 3 mostra a nuvem de assuntos separada por campus. Quanto maior a fonte da palavra, maior a ocorrência da mesma em produções científicas. 
Com a análise de frequência de palavras-chave, percebe-se que o maior número de pesquisas do IFTM está nas áreas de Ensino, Educação, Agrárias e Alimentos.

Figura 2 - Nuvem representando as palavras-chave mais utilizadas em produções científicas dos docentes do IFTM

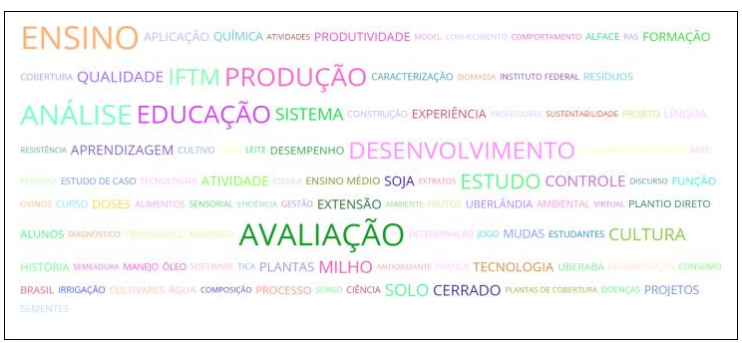

Fonte: Elaborado pelos autores (2020).

Analisando separadamente por campus (Figura 3), nota-se que a palavra Ensino é a que mais se destaca. No campus Ituiutaba, podemos citar algumas palavras: história, produção, sorvete, educação ambiental, resíduos e cinema.

No campus Paracatu, podemos citar palavras interessantes, tais como: espaços públicos, linguagem, planejamento urbano e a palavra Paracatu (mostrando que pesquisas aplicadas são muito utilizadas no campus). No campus Patos de Minas percebemos que a língua inglesa é tema de várias pesquisas. Em Patrocínio destacamos as seguintes palavras: autômatos celulares, gestão de pessoas, robótica e análise do discurso. No campus Uberaba a produtividade voltada para a área de agrárias é muito pesquisada. No campus Uberaba Parque Tecnológico, pesquisas com jogos e sistemas de computador são bem presentes. No campus Uberlândia destacam-se assuntos voltados para produção, qualidade e desenvolvimento na área de agrárias.

Por fim, o campus Uberlândia Centro destaca-se a educação e tecnologia. No campus Campina Verde não está contextualizado na Figura 3 devido ao número pequeno de docentes e ao baixo número de produções científicas.

\subsection{Análise da formação acadêmica}

Nesta etapa, analisou-se a formação acadêmica do corpo docente do IFTM, conforme Figura 4. Ao analisar os 544 docentes do IFTM, observa-se que as formações com maior número de docentes são: 66 em Letras, 54 em Agronomia, 48 em Engenharia Elétrica, 46 em Ciência da Computação, 43 em Administração, 39 em Matemática, 37 em Química e 35 em Ciências Biológicas.

\subsection{Consulta de docentes por assunto}

Mediante a plataforma web (OBSERVATÓRIO IFTM, 2019) desenvolvida neste trabalho, é possível procurar docentes utilizando como parâmetro de busca temas de pesquisa. Além disso, com o objetivo de identificar potenciais grupos de pesquisa com docentes em diversos campi, escolhemos 10 palavras-chave muito utilizadas no IFTM e contabilizamos o número de docentes que possuem produção científica relacionada com estas palavras.

$\mathrm{O}$ resultado deste experimento encontra-se no Quadro 1. Este experimento mostrou que nos 10 assuntos escolhidos existiam docentes de diferentes campi, viabilizando a possibilidade na formação de grupos de pesquisa multicampi, podendo assim aumentar a potencialidade da pesquisa. 
Análise do perfil de docentes...

65

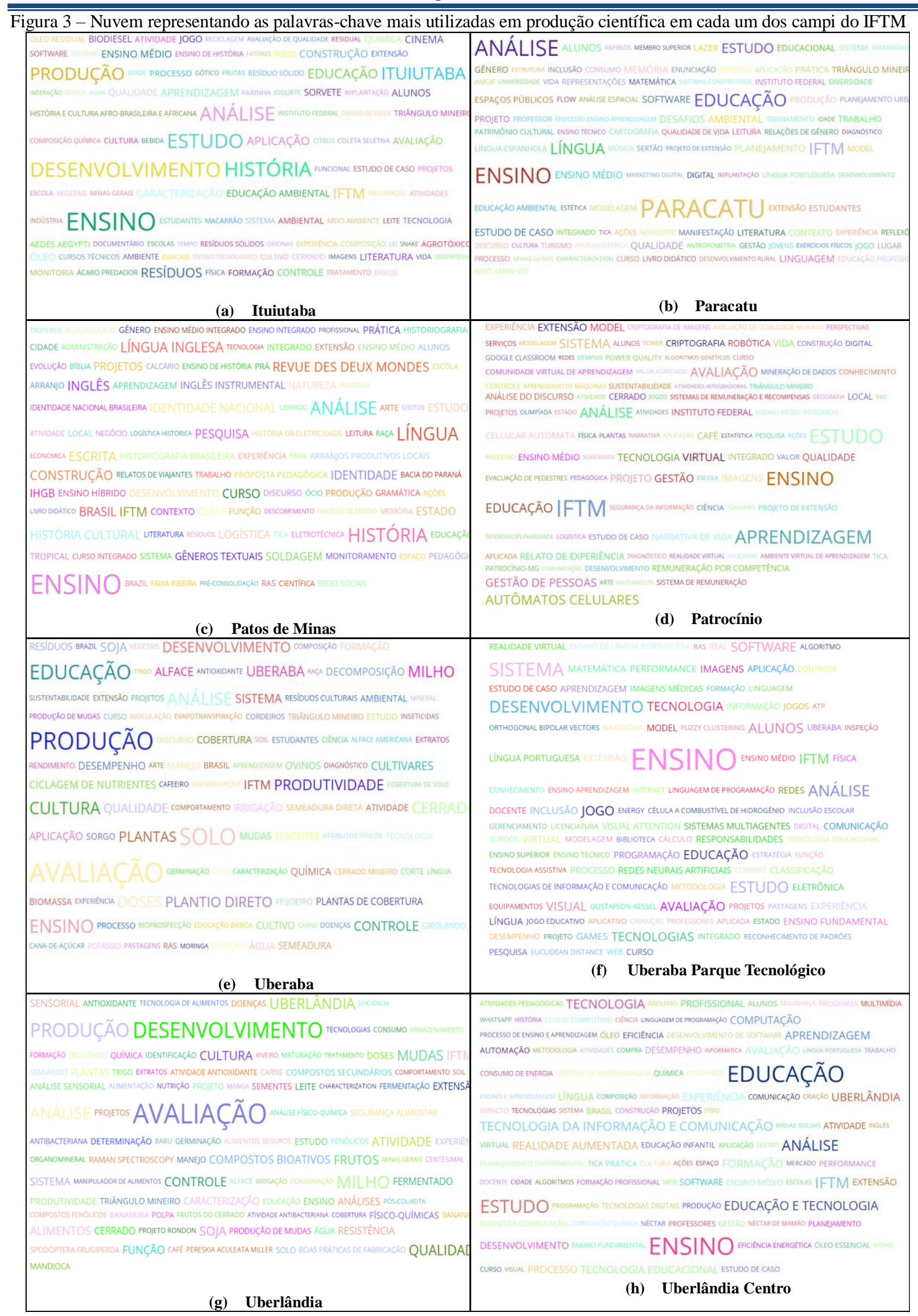

Fonte: Elaborado pelos autores (2020). 
Figura 4 - Formação acadêmica do corpo docente do IFTM

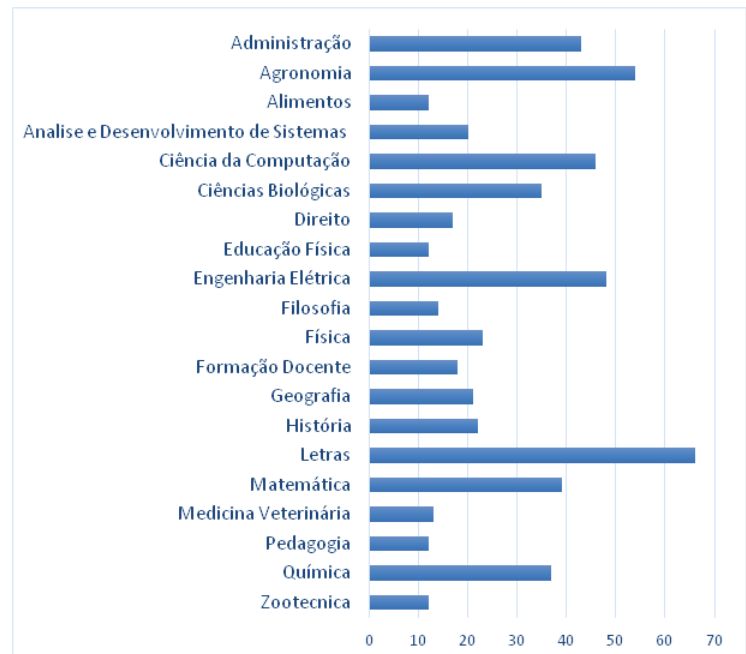

Fonte: Elaborado pelos autores (2020).

Quadro 1 - Quantidade de docentes por assunto pesquisado

\begin{tabular}{|l|c|}
\hline \multicolumn{1}{|c|}{ Palavra-chave } & Número de docentes \\
\hline Milho & 30 \\
\hline Soja & 33 \\
\hline Solo & 28 \\
\hline Irrigação & 12 \\
\hline Sementes & 24 \\
\hline Ensino & 114 \\
\hline Jogo(s) & 23 \\
\hline Língua inglesa & 10 \\
\hline História & 19 \\
\hline Inteligência Artificial & 7 \\
\hline
\end{tabular}

Fonte: Elaborado pelos autores (2020).

\section{Considerações finais}

Com os resultados apresentados neste trabalho, verificou-se que os currículos dos docentes disponíveis na Plataforma Lattes podem ser usados para mapear os perfis dos docentes e encontrar os temas de pesquisas científicas mas discutidos em cada campus do IFTM.

Este trabalho demonstrou a possibilidade de docentes de campus distintos trabalharem em um mesmo grupo de pesquisa. Desta maneira, o discente poderá ser contemplado em um único trabalho científico, com orientações de diferentes docentes pertencentes a grupos de pesquisa multicampi.
Durante os experimentos, constatou-se que alguns temas são comuns a quase todos os campi, tais como: Ensino, Educação, Agrárias e Alimentos. Já temas mais específicos, tais como: Gestão de Pessoas, Autômatos Celulares e Inteligência Artificial acontecem em poucos campi devido ao perfil dos docentes do IFTM.

Ao analisarmos a formação dos docentes, conclui-se que o IFTM tem uma variabilidade muito grande, aumentando assim a diversidade das pesquisas. Com isso, trabalhos como este são fundamentais para auxiliar docentes e discentes nas atividades de pesquisa.

\section{Referências}

Conselho Nacional de Desenvolvimento Científico e Tecnológico $(\mathrm{CNPq})$ Sobre a plataforma Lattes. 2019. Disponível em: 〈http://lattes.cnpq.br/>

CORRÊA, Tiago Silva; SUZUKI, Manuella Barbosa; CINTRA, Paulo Roberto; COSTA, Luzia Sigoli Fernandes. O fim do scriptLattes? Uma análise de suas funcionalidades, alternativas para o presente e perspectivas para o futuro. Revista do EDICC, v. 3, n. 3, p. 138-148, 2017.

DEMO, Pedro. Pesquisa: princípio científico e educativo.14. ed. São Paulo: Cortez, 2011.

DIGIAMPIETRI, Luciano Antonio; MENACHALCO, Jesús P.; PÉREZ-ALCÁZAR, José J.; TUESTA, Esteban F.; DELGADO, Karina V.; SILVA, Gabriela S.; MUGNAINI, Rogério.; LIMA, Jamison J. S. Extração, caracterização e análises de dados de currículos lattes. Revista Eletrônica de Sistemas de Informação, v. 14, n. 2, p. 1-24, 2015.

FÓRUM DE PRÓ-REITORES DE EXTENSÃO DAS UNIVERSIDADES PÚBLICAS BRASILEIRAS (FORPROEX). Indissociabilidade ensino-pesquisa-extensão e a flexibilização curricular: uma visão da extensão. Porto Alegre: UFRGS; Brasília: MEC, 2006.

INSTITUTO FEDERAL DE EDUCAÇÃO, CIÊNCIA E TECNOLOGIA DO TRIÂNGULO MINEIRO (IFTM). Instituto Federal de Educação, Ciência e Tecnologia do Triângulo Mineiro. 2019. Disponível em: 〈http://www.iftm.edu.br>

INSTITUTO FEDERAL DE EDUCAÇÃO, CIÊNCIA E TECNOLOGIA DO TRIÂNGULO MINEIRO (IFTM). Plano de Desenvolvimento Institucional. 2019b. Disponível em: <http://www.iftm.edu.br/pdi/> 
MACCARI, Emerson Antonio; KNIESS, Claudia Terezinha; FERRAZ, Renato Ribeiro Nogueira. Organização e Gestão de Pós-Graduação por Meio da Ferramenta Computacional Scriptlattes. MultiScience Research (MSR), v. 2, n. 1, p. 113-142, 2019.

NIGRO, Carolina Alencar; FERRAZ, Renato Ribeiro Nogueira; QUONIAM, Luc; ALVES, Wonder Alexandre Luz. Uso da ferramenta computacional Scriptlattes-Script sucupira na prestação de contas anual e quadrienal a capes por um programa de Pós-graduação Stricto sensu em Direito. Informação \& Informação, v. 21, n. 1, p. 131-158, 2016.

${ }^{\mathrm{i}}$ Disponível em: 〈http://lattes.cnpq.br $>$

ii A ferramenta scriptLattes está disponível em $<$ http://scriptlattes.sourceforge.net/>

iii O Sistema Eletrônico do Serviço de Informações ao Cidadão (e-SIC) permite que qualquer pessoa, física ou jurídica, encaminhe pedidos de acesso à informação, acompanhe o prazo e receba a resposta da solicitação realizada para órgãos e entidades do Executivo Federal. Disponível em: $\langle$ https://esic.cgu.gov.br/sistema/site/index.aspx $>$

${ }^{\text {iv }}$ Disponível em: <https://pt-br.reactjs.org/> Disponível em: $\langle$ https://www.primefaces.org/primereact/\#/> ${ }^{v i}$ Disponível em: <https://material-ui.com/> vii Disponível em: <https://docs.microsoft.com/ptbr/aspnet/core/>
OBSERVATÓRIO IFTM. 2019. Disponível em: <https://obsiftm.midi.upt.iftm.edu.br/>

OLIVEIRA, E.; BERMEJO, P. D. S.; KERN, V. M. GeraLattes: extração de informação gerencial de currículos de pesquisadores usando XML. In: Workshop de Computação da Região Sul (WorkCompSul 2004), 1., Florianópolis. Anais... UNISUL, 2004.

SOMOS-UFMG. Plataforma Somos UFMG. 2020. Disponível em: 〈http://www.somos.ufmg.br〉 